ARTICLE

Received 10 Dec 2015 | Accepted 4 Jul 2016 | Published 9 Aug $2016 \quad$ DOl: 10.1038/ncomms12453

\title{
Local adaptation of a bacterium is as important as its presence in structuring a natural microbial community
}

Pedro Gómez ${ }^{1,2}$, Steve Paterson ${ }^{3}$, Luc De Meester ${ }^{4}$, Xuan Liu $^{3}$, Luca Lenzi ${ }^{3}$, M.D. Sharma ${ }^{5}$, Kerensa McElroy ${ }^{6}$ \& Angus Buckling ${ }^{1}$

Local adaptation of a species can affect community composition, yet the importance of local adaptation compared with species presence per se is unknown. Here we determine how a compost bacterial community exposed to elevated temperature changes over 2 months as a result of the presence of a focal bacterium, Pseudomonas fluorescens SBW25, that had been pre-adapted or not to the compost for 48 days. The effect of local adaptation on community composition is as great as the effect of species presence per se, with these results robust to the presence of an additional strong selection pressure: an SBW25-specific virus. These findings suggest that evolution occurring over ecological time scales can be a key driver of the structure of natural microbial communities, particularly in situations where some species have an evolutionary head start following large perturbations, such as exposure to antibiotics or crop planting and harvesting.

\footnotetext{
${ }^{1}$ ESI and CEC, Biosciences, University of Exeter, Penryn Campus, Cornwall TR10 9FE, UK. ${ }^{2}$ CEBAS-CSIC, Campus Espinardo, 30100 Murcia, Spain. ${ }^{3}$ Institute of Integrative Biology, University of Liverpool, Liverpool L69 7ZB, UK. ${ }^{4}$ Laboratory of Aquatic Ecology, Evolution and Conservation, KU Leuven, 3000 Leuven, Belgium. ${ }^{5}$ CEC, Biosciences, University of Exeter, Penryn Campus, Cornwall TR10 9FE, UK. ${ }^{6}$ Common wealth Scientific and Industrial Research Organisation (CSIRO), Canberra GPO Box 1700, Australia. Correspondence and requests for materials should be addressed to P.G. (email: pglopez@cebas.csic.es).
} 
C ommunity structure changes in response to environmental perturbation because some species perform better under the novel conditions than others ${ }^{1}$. In parallel with recent theory ${ }^{2}$, there is a growing body of empirical work that suggests contemporaneous evolution (evolution occurring on the same time scale as ecological changes) may contribute to these changes in community composition ${ }^{3-7}$. Studies using simplified laboratory communities of microbial organisms ${ }^{8-11}$ show that contemporaneous evolution can affect interactions within- and between-trophic levels, and historical effects of microevolution have been shown to affect subsequent community structure in more complex settings ${ }^{12-15}$.

A number of studies have attempted to determine the relative impact of contemporaneous evolution versus ecological variables on community structure ${ }^{7,14,16}$. For example, local adaptation of Daphnia magna was found to be as important as the presence of predatory fish in driving the structure of zooplankton communities in mesocosms ${ }^{7}$. However, while this work provides important information on the relative importance of specific environmental variables and adaptation to these variables, a more general way to estimate importance of local adaptation is to measure its effect relative to the presence of the evolving species per se.

Here, we concurrently determine how both the presence of a focal species and local adaptation of this species affect the structure of a natural soil (compost) microbial community, and consequently the relative importance of contemporaneous evolution to species presence per se. As a focal species, we use the bacterium Pseudomonas fluorescens SBW25 (ref. 17), which we have previously shown to be able to adapt rapidly to a sterile compost environment by diversifying into resource niche specialists ${ }^{18}$. We estimate the ecological impact of $P$. fluorescens SBW25 by comparing how its presence versus absence affects the natural community structure in compost microcosms propagated over 60 days. Likewise, we estimate the effect of contemporaneous evolution by comparing how SBW25 that had been adapted to the compost microcosms (for 48 days) and ancestral SBW25 affect community structure. To increase the potential importance of local adaptation, we propagated microcosms at an elevated temperature $\left(26^{\circ} \mathrm{C}\right)$, representing a novel environment for both the compost-dwelling community (novel temperature) and sugar beet-associated SBW25 (ref. 17; novel temperature and substrate). Finally, we explore the robustness of our findings by determining how an additional ecologically relevant selection pressure may affect the importance of species presence and additional local adaption affects community structure. We have previously shown that a highly SBW25-specific bacteriophage, SBW25 $\$ 2$ (ref. 19), impose strong selection on SBW25 in soil ${ }^{20,21}$, as apparent from both density reductions and resistance evolution, hence we inoculated this virus into additional microcosms containing ancestral and locally adapted SBW25.

We found that contemporaneous adaptive evolution of a single species can play as great a role as species presence in structuring natural microbial communities. Crucially, we detected large differences in community composition between treatments, with the effect of adaptation as great as the effect of the presence of SBW25 per se. These results provide an experimental verification of evolutionary priority effects theory in natural microbial communities, with a particular relevance for understanding how natural microbial communities become established, and hence the impact they may have on ecosystem function and host health.

\section{Results}

Local adaptation of Pseudomonas fluorescens SBW25 to compost. We first determined whether the six SBW25 populations that had been previously cultured in sterile compost under equivalent abiotic conditions as used here ${ }^{18}$ had adapted to the compost environment. To this end, the relative fitness of SBW25 populations was measured by competing them against their lacZ-marked ancestor, after confirming no difference in competitive fitness of the unmarked and marked ancestor (one sample $t$-test; $n=6 ; t=1.4 P>0.6$ ). Large increases in competitive fitness relative to the ancestor were detected when measured in both the presence and absence of the natural communities (one sample $t$-test; $t=0.03 P<0.01$ in both cases, Fig. 1). To confirm that these phenotypic changes were correlated with genetic changes, we sequenced a single clone from each of the six populations. Non-synonymous mutations were detected in all populations (a list of either single-nucleotide polymorphisms or small insertions and deletions can be found as Supplementary Data 1). However, given that most mutated genes only occurred in single clones, and the few parallel changes that did occur affected genes of unknown functions, it is difficult to infer links between genomic and phenotypic changes. Unambiguous parallel changes occurred in the PFLU1093 and PFLU4198 genes, which were disrupted by insertions that led to frame-shifts. These genes encode a putative fimbrial usher protein and a putative sensory box GGDEF/EAL domain-containing protein that, in particular, are associated with the biogenesis of pili and the phosphorylation catalysis in response to detection of a chemical ligand or change in environment that initiate a change in cell activity, respectively.

Changes in the population densities. We determined the population densities of SBW25, SBW25\$2 and members of the natural microbial community that could be cultured in vitro under similar conditions to SBW25 bacterial communities after 30 and 60 days, ensuring ecological changes were measured over a comparable time scale to the 48 days of SBW25 evolution. Locally adapted SBW25 reached on average approximately three times greater mean population density than ancestral SBW25 in the presence of the natural community (Fig. 2a, Linear Mixed Effects Models (LMM), $F_{1,20}=82.2, P<0.001$ ), providing a clear mechanism by which local adaptation might affect community composition. Phages reduced the density of the SBW25

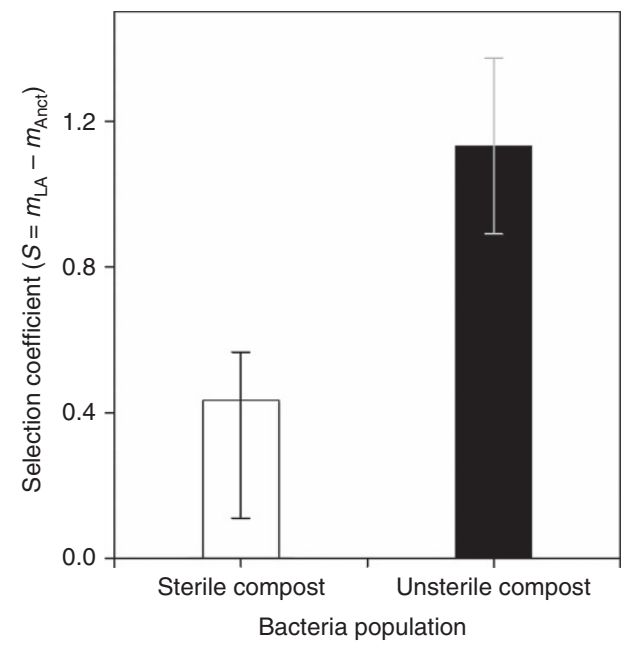

Figure 1 | Fitness of locally adapted SBW25. Mean ( \pm s.e.m.) selection coefficient of evolved SBW25 (after 48 days cultivation in sterile compost at $26^{\circ} \mathrm{C}$ ) determined by competing against its ancestor for 5 days in both sterile and unsterile compost. Note that selection coefficient $(S)$ was calculated here by the difference between the estimated Malthusian parameter of the locally adapted SBW25 population $\left(m_{\mathrm{LA}}\right)$ and the ancestral SBW25-lacZ strain $\left(m_{\text {Anct }}\right)$, where a value of zero indicates equal fitness. 
a

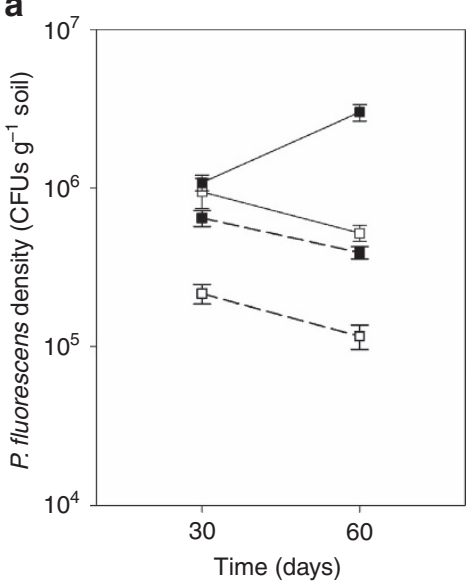

b

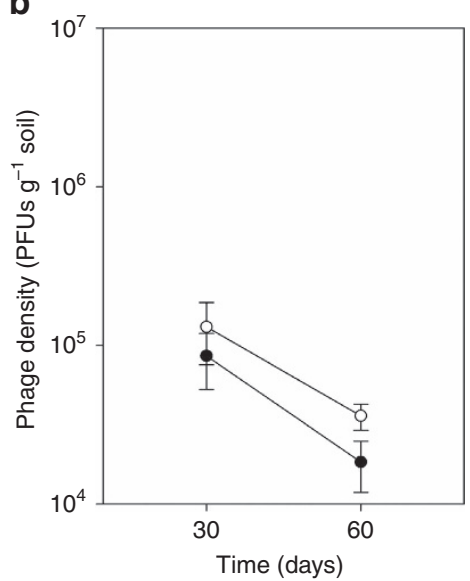

C

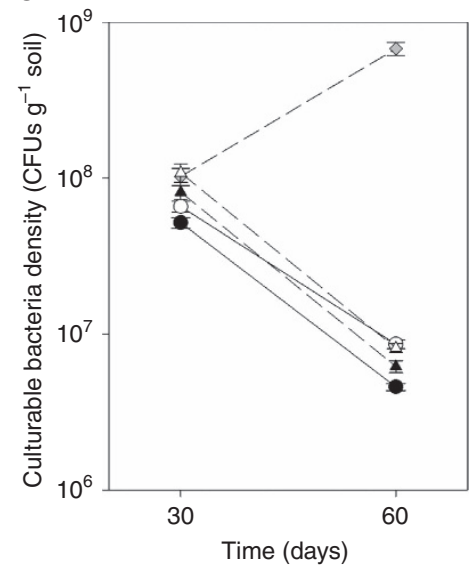

Figure 2 | Bacterial population densities in the natural microbial community. (a) Mean densities ( \pm s.e.m.) of the six replicates of ancestral (white square) and locally adapted (black square) P. fluorescens SBW25 (CFUs ${ }^{-1}$ soil) in the presence of the natural microbial community (solid line) and the presence of

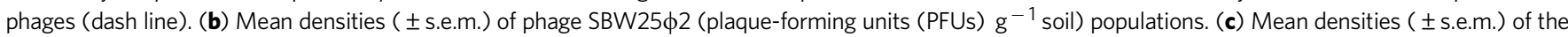
six replicates of the culturable bacteria (CFUs g ${ }^{-1}$ soil) in the natural microbial community with no SBW25 (grey diamond), ancestral SBW25 (white circle, white triangle) and locally adapted SBW25 (black circle, black triangle) in the absence (solid line) and presence (dashed line) of phages.

approximately 4 -fold (LMM, $\left.F_{1,20}=123.5, P<0.001\right)$, with no differential reduction between ancestral and locally adapted SBW25 (Fig. 2a, SBW25 treatment by phage: $F_{1,20}=0.75$, $P=0.4$ ). Phage densities were not significantly different when co-evolving with ancestral or locally adapted SBW25 (Fig. 2b, LMM, $\left.\quad F_{1,10}=3.8, \quad P=0.08\right)$. Note that phage densities significantly decreased from day 30 to 60 (Fig. 2b, LMM, $F_{1,11}=30.3, P<0.001$ ), while SBW25 densities typically decreased between these time points, except locally adapted SBW25 in the absence of phages increased (Fig. 2a, LMM, time by phage by SBW25 treatment: $F_{1,20}=5.5, P=0.3$ ).

The density of culturable bacteria that grew under the same culture media on which SBW25 thrives was reduced approximately 10 -fold by the presence of SBW25 in the absence of phages (Fig. 2c; LMM, $F_{2,15}=682, P<0.001$ ). There was a further approximately $50 \%$ reduction resulting from the presence of locally adapted SBW25 (Fig. 2c; LMM, $F_{1,20}=25.88, P<0.001$ ), while the presence of phages increased the density of culturable bacteria by approximately $50 \%$ (Fig. 2 c; LMM, $F_{1,20}=16.88$, $P<0.001$ ), with no interaction between phages and whether SBW25 had been locally adapted or not (Fig. 2c, LMM, $\left.F_{1,20}=1.26, P=0.27\right)$. Densities of culturable bacteria decreased through time in the presence of SBW25, but increased in the absence of phages (Fig. 2c, LMM, time by SBW25 treatment: $\left.F_{1,27}=326, P<0.001\right)$. In summary, these data show that while locally adapting SBW25 had a significant effect on the density of culturable bacteria, this effect size was marginal (approximately $5 \%$ ) compared with that of the presence of SBW25 per se.

Changes in the bacterial community structure. Using a simple culture-independent method (amplicon sequencing of a variable region of $16 \mathrm{~S} \mathrm{rDNA}^{22}$ ) we detected high levels of diversity within each of the bacterial communities, with approximately $10^{3}$ distinct operational taxonomic units (OTUs) per community. Despite this high level of diversity, we detected large differences in the relative frequency of different OTUs between treatments (no phages: no SBW25, ancestral and locally adapted; phages: ancestral and locally adapted SBW25) and time points (Figs 3a and 4; all treatments and time points differed; PERMANOVA $P<0.01$ for all pairwise comparisons). Regardless of the presence of phages, differences in community composition (based on weighted UniFrac distances) between the locally adapted and ancestral SBW25 treatments were of a similar magnitude to those between the ancestral and no SBW25 treatments (Fig. 3a,b). There was a tendency for phages to reduce the impact of both SBW25 treatments on community structure (Fig. 3b), but phages had a much greater impact on community structure-imposed changes caused by ancestral than locally adapted SBW25 (Fig. 3c).

Although there were many differences in the frequency of specific groups of bacteria between treatments within time points, many of these differences were reversed between time points (Fig. 3d), hence removing any net effects between treatments. The notable exception was the Bacillales order, a common sporeforming group of soil bacteria, which had elevated frequencies in the presence of locally adapted SBW25 in both the presence and absence of phages, (Fig, $3 \mathrm{~d}$; two-sample $t$-test, $P<0.001$ for all comparisons). Note that the frequency of the order (or any lower taxonomic unit) to which SBW25 belongs, the Pseudomonadales, was not consistently elevated following the addition of ancestral or locally adapted SBW25, demonstrating that differences in community composition were not driven by the direct effect of the addition of SBW25.

Comparison of the community diversity metrics. There was no overall difference in between-community diversity (betadiversity) across time and treatments (Fig. 5, permutation test; $P=0.09)$. Moreover, there was also no overall difference in mean within-community diversity between any treatment (Fig. 6; LMM, $F_{4,26}=1.72, P=0.17$ ) or between time points (Fig. 6, LMM, $\left.F_{2,26}=2.11, P=0.14\right)$.

\section{Discussion}

While contemporaneous evolution of a focal species has been shown to affect the structure of natural communities, its importance relative to purely ecological effects of the presence of the species has not been tested directly. Here, we show that local adaptation of a focal strain of bacteria ( $P$. fluorescens SBW25) for a matter of weeks influenced the subsequent structure of a natural compost microbial community as much as the presence of that strain per se. Local adaptation, however, had a much smaller relative effect than species presence on community population dynamics, and neither treatments significantly affected community diversity metrics. Moreover, the relative impact of local adaptation was similar regardless of 
a



b

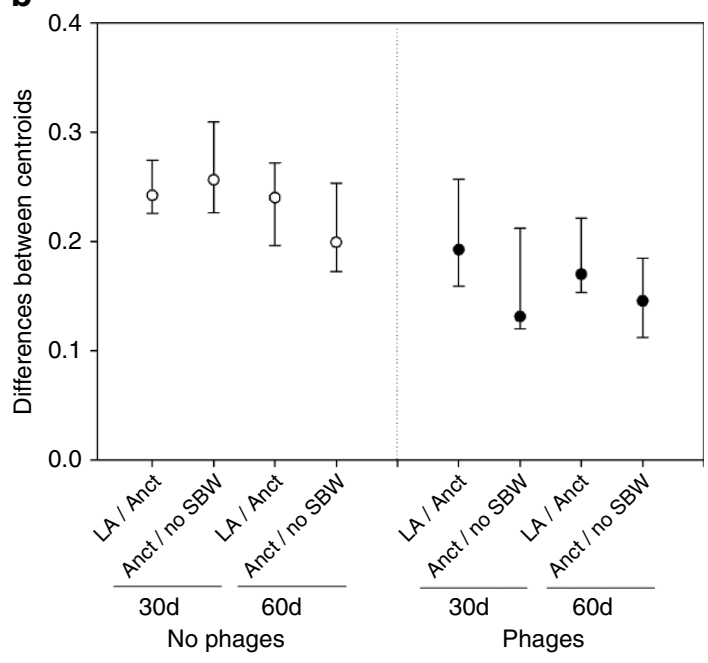

C

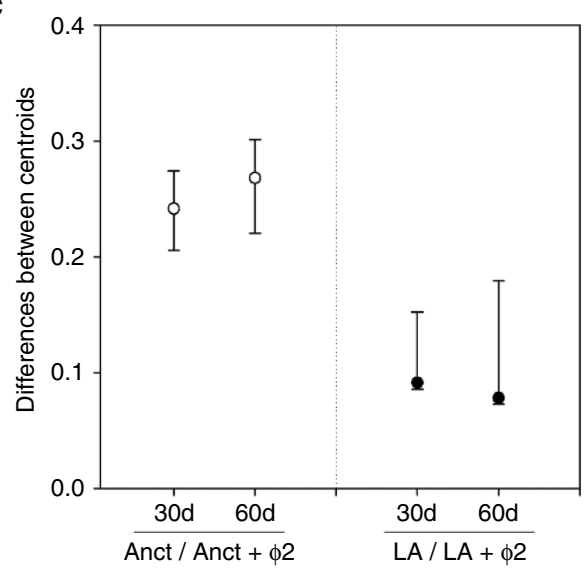



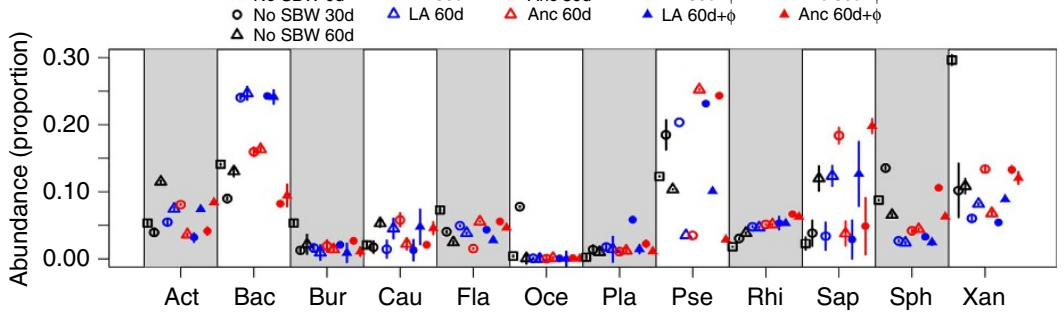

$\overline{\text { Anct / Anct }+\phi 2}$

Figure 3 | Changes in community composition. (a) Principal Coordinate Analysis (PCoA) plot of communities based on UniFrac distances. The percentage of variation explained is shown on each axis (calculated from the relevant eigenvalues). Replicates within treatments and time points have similar community compositions, while there are notable differences between treatments and time points. (b) Points show mean ( $95 \% \pm$ confidence intervals $(\mathrm{Cls})$ ) distances between treatment centroids at 30 and 60 days in the absence (open circles) and presence (closed circles) of phages. These centroids are the mean position of all the points in all coordinate directions from the PCoA plot, and their differences reflect the effect of adaptation and species presence on community change. The difference between ancestral and locally adapted treatments shows the magnitude of the effect of adaptation, while difference between presence and absence of SBW25 shows the magnitude of effect of species presence. (c) Points show mean (95\% \pm Cls) distances between treatment centroids to show how phages $(+\phi 2)$ affect community structure in the presence of either ancestral or evolved SBW25. (d) Common bacterial orders across treatments ( $>5 \%$ in at least one treatment-time point combination). Note consistently high frequency of Bacillales (Bac) across time in evolved SBW25 treatment. Act, Actinomycetales; Bur, Burkholderiales; Cau, Caulobacterales; Fla, Flavobacteriales; Oce, Oceanospirillales; Pse, Pseudomonadales; Rhi, Rhizobiales; Sap, Saprospirales; Sph, Sphingobacteriales; Xan, Xanthomonadales. Square represents day 0, circle represents day 30, triangle represents day 60; black represents No SBW25; blue represents locally adapted (LA), red represents ancestral (Anct); open symbols indicate no phage, closed symbols indicate with phage $(+\phi)$.

the presence of an SBW25-specific virus that resulted in SBW25 density reductions comparable to the increase in density resulting from local adaptation, suggesting our findings are likely to be robust in the face of additional strong selection pressures.

Although we cannot explain the specific changes in community structure caused by SBW25, general niche theory ${ }^{23}$ would suggest that bacteria that share a similar ecological niche to SBW25 are likely to be negatively affected by its presence and more so if it is locally adapted. Consistent with this view, we found the density of bacteria that are favoured by the same high nutrient culture media on which SBW25 thrives, to be lower in the presence versus absence of SBW25 and lower still when SBW25 had been locally adapted. Moreover, their density was increased when SBW25 density was reduced by the phage. Mechanistically, the production of antimicrobials ${ }^{24}$ is a key trait that may link SBW25 adaptation with altered community composition, since these antimicrobial products play important roles in interactions between soil-associated Pseudomonads and other members of the community ${ }^{25}$.

In contrast to the effects on community composition, it is notable that the effect of local adaptation had relatively little impact on other community characteristics. First, the density of readily culturable bacteria was little affected compared with the effect of the presence of SBW25 per se, highlighting the unpredictability of the cascading effects of small changes in the frequency of specific taxa across the whole community. Second, the lack of effect on within- and between-population diversity alongside the clear difference in community composition emphasize the role of deterministic (niched-based) processes, in structuring these compost communities ${ }^{26}$. 




Figure 4 | Differences in community composition. Principal Coordinate Analysis (PCoA) based on Bray-Curtis dissimilarity of bacterial orders between communities. PCA loadings for orders playing key roles in determining community differences are shown for comparison as arrows: Bac, Bacillales; Pse, Pseudomonadales; Sap, Saprospirales; Sph, Sphingobacteriales; Xan, Xanthomonadales. Square represents day 0, circle represents day 30, triangle represents day 60; black represents No SBW25; blue represents locally adapted (LA), red represents ancestral (Anc); open symbols indicate no phage, closed symbols indicate with phage $(\phi 2)$.

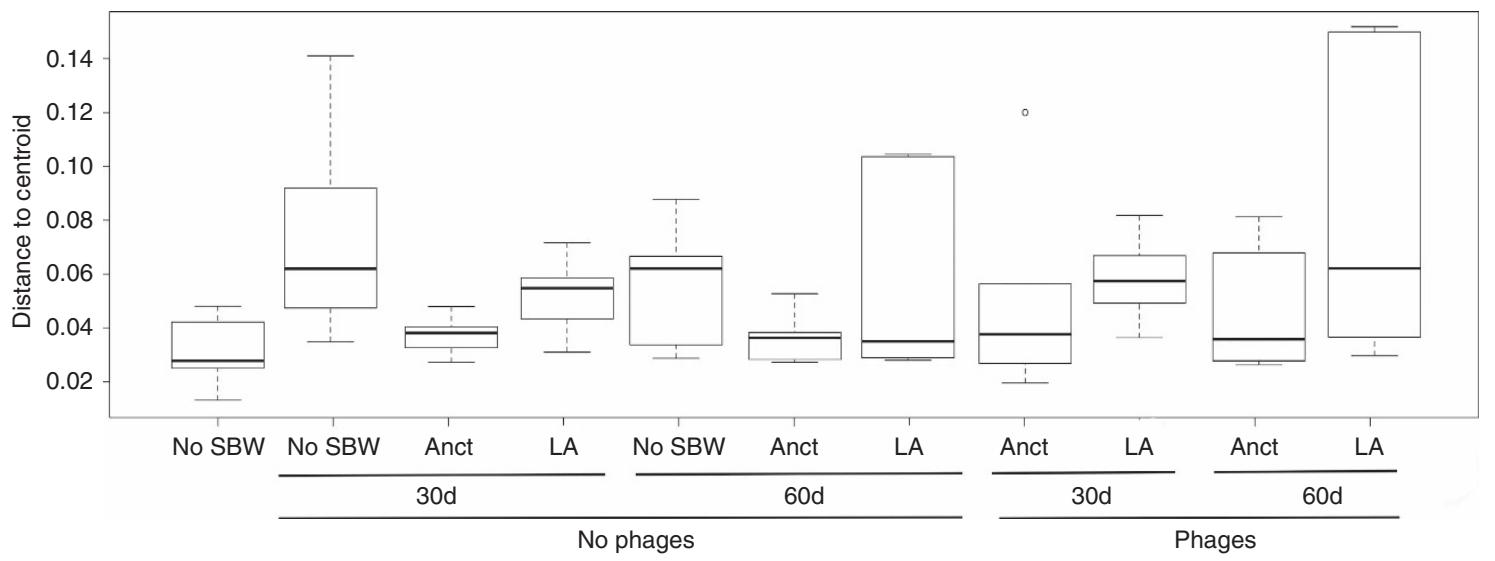

Figure 5 | Beta diversity. Box plot representation of the values of the distances to centroid for treatment-time point combinations; No SBW25, ancestral (Anct) and locally adapted (LA) treatments at 30 and 60 days in the absence and presence of phages, based on UniFrac distances. The box plots show medians (horizontal line in box), 25 and $75 \%$ quartiles, and max/min values, outliers marked as circles.

The frequency of bacterial OTUs (that is, taxa detected from sequencing) varied between treatments and time points, often in the opposite way. However, one consistent finding across time is that SBW25 (especially when locally adapted) increased the frequency of Firmicutes. We do not know why these particular changes in the community occurred, but these findings are consistent with recent work on mouse gut microbial communities where large increases in the frequency of Firmicutes (the phylum to which the Bacillales belong) resulted from upregulation of secondary metabolites by Escherichia coli ${ }^{27}$.

Although our data suggest that the density of a given species is a predictor of its impact on the community as a whole, it is by no means the complete explanation. For example, we found that the presence of phages had a tendency to reduce the impact SBW25 had on the community, but this impact was much less for locally adapted than ancestral SBW25, despite a comparable phageimposed density reduction. This effect of phages may be because the phages also result in selection for resistance and other correlated traits $^{20,28,29}$, and these consequences may have differed between ancestral and locally adapted populations. It may also be that ancestral and locally adapted SBW25 interact with other co-occurring species in different ways regardless of the impact of phages. Consistent with this view, the locally adapted SBW25 population had adaptively diversified, with different genotypes 


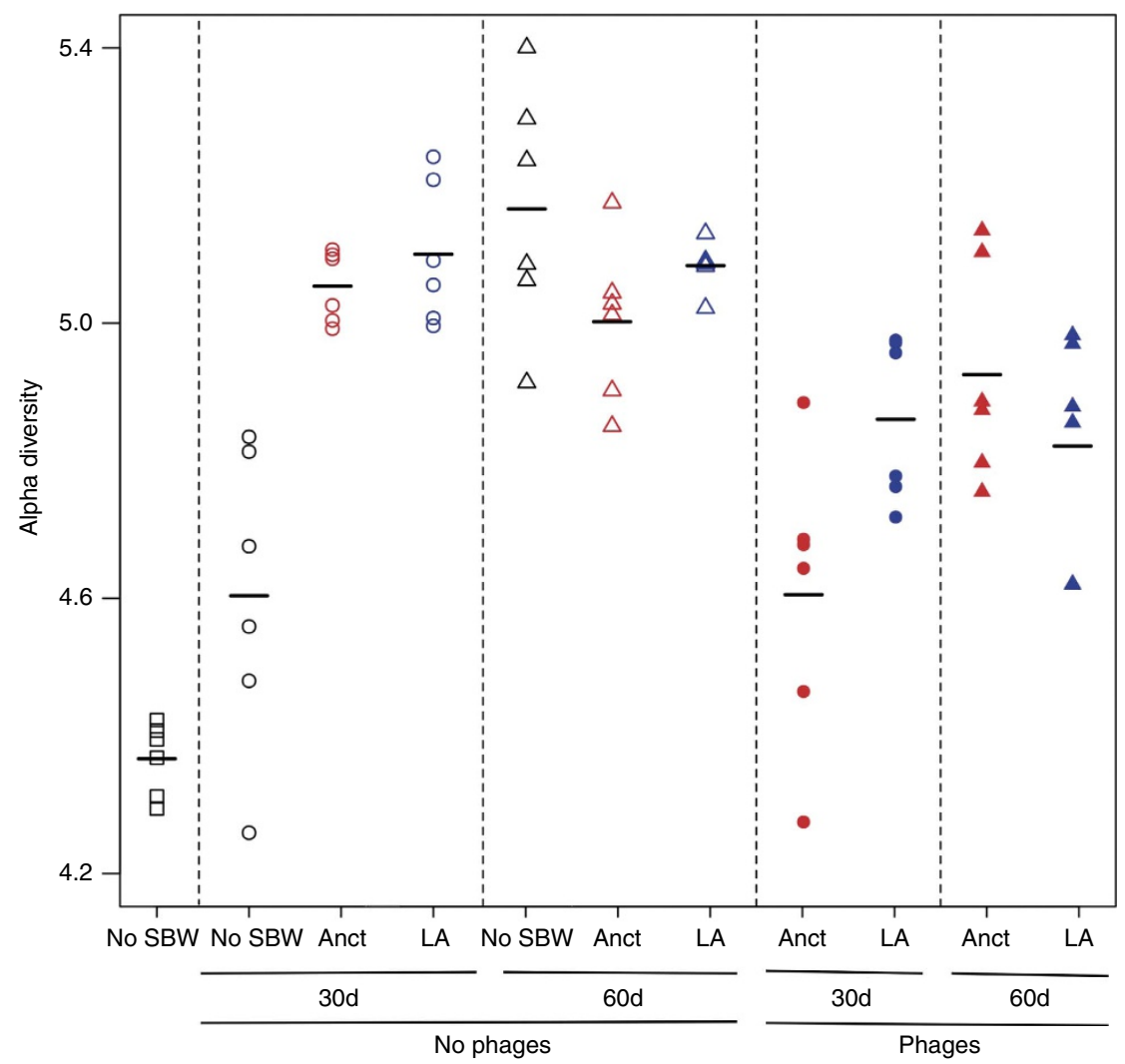

Figure 6 | Shannon diversity index. Representation of the within-population diversity values of the community from No SBW25, ancestral and locally adapted treatments at 30 and 60 days in the absence and presence of phages. Square represents day 0, circle represents day 30 , triangle represents day 60; black represents No SBW25; red represents ancestral (Anct); blue represents locally adapted (LA); open symbols indicate no phage, closed symbols indicate with phage $(+\phi)$.

having different catabolic profiles that suggest the ability to use different resource ${ }^{17}$. The importance of genotypic diversity on community structure has been highlighted in other systems. For instance, increased genotypic diversity of the perennial plant Solidago altissima had a major impact on arthropod species diversity $^{30}$.

Our experiment was specifically designed to compare the effect of adaptive contemporaneous evolution with species presence. Of course, in many contexts, single species will not evolve in isolation, but instead species would be simultaneously (co-)evolving, and it is unclear whether this would increase or decrease the relative importance of contemporaneous evolution in determining community structure. Our results do, however, have clear ecological relevance for colonization following environmental perturbations. Colonization order is a key factor determining community structure because early colonists can reach high densities and dominate resources before other species arrive ('priority effects') ${ }^{31-34}$. Our work is consistent with previous theoretical and empirical studies showing that evolution occurs on ecological time scales ${ }^{3-15}$, and hence, early colonization can further enhance priority effects by allowing species to become locally adapted ${ }^{35-39}$. More specifically, we show that priority effects resulting from local adaptation can be equally important as ecological priority effects driven by the mere presence of a species. In summary, contemporaneous evolution is likely to play an important role in the structure and function of natural microbial communities particularly in cases where existing communities are strongly perturbed, including the plant rhizosphere in agricultural systems and the gut microbiome exposed to antibiotics ${ }^{40}$.

\section{Methods}

Bacterial inoculation into soil-microcosms and sampling. We had previously cultured and frozen six replicates of gentamicin-resistant $P$. fluorescens SBW25 in compost microcosms for 48 days under the same abiotic conditions used for the current experiment $t^{28}$. These populations were mixed, grown overnight at $28^{\circ} \mathrm{C}$ in King's Media B (KB) ${ }^{41}$ and then $\sim 10^{6}$ colony-forming units (CFUs) $\mathrm{g}^{-1}$ of soil were inoculated into 12 replicate sterile soil-microcosms. In parallel, 12 microcosms were inoculated with the same density of overnight cultures of the gentamcin-resistant SBW25 ancestor. These soil-microcosms were based on polypropylene trays $(10 \times 10 \mathrm{~cm})$ containing $100 \mathrm{~g}$ of twice-autoclaved compost (Verve Multi-Purpose Compost, UK) soil ${ }^{18,20}$. The next day, half the microcosms (six per treatment) were inoculated with $5 \mathrm{ml}$ of M9 salt solution $\left(12.8 \mathrm{gl}^{-1}\right.$ $\mathrm{Na}_{2} \mathrm{HPO}_{4}-7 \mathrm{H}_{2} \mathrm{O} ; 3 \mathrm{gl}^{-1} \mathrm{KH}_{2} \mathrm{PO}_{4} ; 0.5 \mathrm{gl}^{-1} \mathrm{NaCl} ; 0.1 \mathrm{gl}^{-1} \mathrm{NH}_{4} \mathrm{Cl}$ ) containing a suspension $\left(10^{6}\right.$ plaque-forming units) of the virulent bacteriophage SBW25 $\phi 2$ (ref. 19). After 10 days, all microcosms were then inoculated with $3 \mathrm{ml}$ of a soilwash ( $20 \mathrm{~g}$ of soil compost per $100 \mathrm{ml} \mathrm{M} 9$ buffer) containing the resident microbial community ( $\sim 10^{5} \mathrm{CFUs} \mathrm{g}^{-1}$ soil), an extra six microcosms were also inoculated with soil-wash for the no SBW25 treatment ${ }^{20}$. Soil microcosms were placed in an environmental chamber at $26^{\circ} \mathrm{C}$ and $80 \%$ relative humidity, conditions fixed to allow a balanced bacterial growth of the whole community. Soil samples $(2 \mathrm{~g})$ were collected at 0,30 and 60 days ${ }^{18,20,21,42}$, and each soil sample was vortexed for $1 \mathrm{~min}$ with M9 buffer ( $5 \mathrm{mlg}^{-1}$ soil). The resultant soil washes were used to determine P. fluoresncens and culturable bacteria densities by plating onto KB agar supplemented with gentamicin $\left(15 \mu \mathrm{g} \mathrm{ml}^{-1} \mathrm{~KB}\right)$ and $\mathrm{LB}$ media, respectively. Note that we used a 'mark-recapture' approach to follow the ecological and evolutionary dynamics, where there were no culturable bacteria in the compost that were able to grow on gentamicin ${ }^{18,20}$. Soil washes were also used to extract total genomic DNA and identify the bacterial community composition by $16 \mathrm{~S}$ rDNA amplicon sequencing. Note that from each replicate population and time point sampled, a soil-microcosm suspension was stored at $-20^{\circ} \mathrm{C}$ in glycerol solution (20\%).

Relative fitness assay. The relative fitness of the locally adapted $P$. fluorescens SBW25 population was determined by standard 5-day competition experiments ${ }^{43}$ against a marked strain of ancestral SBW25-lac ${ }^{44}$ in soil in the presence and absence of the microbial community. Approximately $10^{7}$ CFUs from both SBW25 strains (overnight cultures grown by shaking at $28^{\circ} \mathrm{C}$ ) were co-inoculated into 24 
sterilized soil-microcosms, half of them containing the resident microbial community. To compare the relative fitness of the locally adapted SBW25 population to the ancestral SBW25-lacZ strain under both conditions, here we used the estimation of selection coefficient $(S)$, which was calculated by the difference between the estimated Malthusian parameter $(m)^{43}$; where $m=\ln \left(N_{\mathrm{f}} / N_{0}\right)$, and $N_{0}$ is the initial and $N_{\mathrm{f}}$ the final bacteria density of the population after 5 days of competition. Bacterial population densities were determined from plating on LB agar containing X-gal $\left(40 \mu \mathrm{g} \mathrm{ml}^{-1}\right)$.

DNA isolation and 16S rDNA amplicon library generation. Total genomic DNA was isolated from each replicate population and time point sampled using a FastDNA spin kit for soil (MP Biomedicals, Solon, OH, USA) following the manufacturer's instructions. For the $16 \mathrm{~S}$ rDNA gene amplicon sequencing, $254 \mathrm{bp}$ conserved fragment from the $\mathrm{V} 4$ hypervariable region was targeted using N501f (5'-AATGATACGGCGACCACCGAGATCTACACTAGATCGCACACTC TTTCCCTACACGACGCTC- $\left.3^{\prime}\right)$ and N701r (5'-CAAGCAGAAGACGGCATAC GAGATTCGCCTTAGTGACTGGAGTTCAGACGTGTGCTC-3') primers and with a pool of indexed primers (sequences can be found in Supplementary Data 2) suitable for multiplex sequencing with Illumina technology 45 . After quantification by fluorometric, $5 \mathrm{ng}$ genomic DNA was mixed with $0.25 \mu \mathrm{l}$ of each $16 \mathrm{~S}$ primer $(10 \mu \mathrm{M})$ and $0.5 \mu \mathrm{l}$ of each of the nested primers $(10 \mu \mathrm{M})$. KAPA amplification mix $(2 \times)$ was used and the final volume was $20 \mu \mathrm{l}$. A negative control of water eluted from the FastDNA spin kit was also included. The samples were amplified at the following conditions: $98^{\circ} \mathrm{C}$ for $2 \mathrm{~s}$ (one cycle), $95^{\circ} \mathrm{C}$ for $20 \mathrm{~s}, 65^{\circ} \mathrm{C}$ for $15 \mathrm{~s}, 72^{\circ} \mathrm{C}$ for $30 \mathrm{~s}$ ( 25 cycles), $72^{\circ} \mathrm{C}$ for $5 \mathrm{~min}$ (one cycle), $4{ }^{\circ} \mathrm{C}$ hold. The samples were then cleaned up using Agencourt Ampure XP beads (Beckman Coulter) at a ratio 1:1. The products were eluted in $12 \mu \mathrm{l} 10 \mathrm{mM}$ Tris $\mathrm{pH}$ 7.5. The samples were analysed by Qubit fluorometry and Bioanalyser. For each protocol, a total of $42 \mathrm{MiSeq}$ sequence libraries were made from seven groups, with six replicates per group.

Sequencing and bioinformatic analysis. Amplicon sequencing was performed by Illumina MiSeq technology at Centre for Genomic Research (University of Liverpool). Each pool of amplicons was sequenced at $2 \times 250$ bp paired-end sequencing with chemisty v2. Sequence data were performed using an adjusted pipeline; Casava v1.8.2 and Cutadapt v1.2.2 were used to perform the basecalling, de-multiplexing and trimming of the indexed reads ${ }^{22,46,47}$. Per sample, 54,000-260,000 filtered read pairs were analysed, and assembled into a single sequence by Flash $^{48}$, and then Qiime v1.8 was used for metagenomic analysis ${ }^{22}$. Clustering sequences at $97 \%$ of similarity generated 1,298 OTUs, the de novo OTU-picking and their quantification was done by using USEARCH ${ }^{49}$ v7.0. Sequences falling below the $97 \%$ similarity threshold for any of the OTUs clusters were removed from further analyses, to act as a filter against potentail artefacts caused by sequencing error. The Greengenes database of ribosomal RNA sequences ${ }^{50}$ v12.8 was used as reference for chimera detection and taxonomy assignments. The taxonomic assignments for each OTU was performed by using Qiime v1.8 and RDP classifier ${ }^{51}$.

Resequencing methods. DNA was extracted from one randomly picked clone from each of the six locally adapted replicas of gentamicin-resistant $P$. fluorescens SBW25 and a clone of ancestral SBW25. Illumina TruSeq libraries were prepared and paired-end sequence generated on an Illumina GAIIx platform. Reads were mapped to the SBW25 reference genome (GenBank NC_012660.1) using bwa (v0.5.9-r16), with local realignment and variant calling (relative to the ancestral SBW25 genome sequenced at the same time) achieved using GATK UnifiedGenotyper (v2.1-13-g1706365) followed by snpEff (v4.1) to assign effects on coding genes.

Statistical analyses. The impact of the different treatments on bacteria densities and within-population (alpha) diversity (Shannon index) during the 60-day experiment was determined using Linear Mixed Effects Models, where density was log-transformed, and treatments and time fitted as fixed effects, and population fitted as a random factor. A one-sample $t$-test was used to determine whether the relative fitness for ancestral and locally adapted SBW25 differed from each other. These analyses were carried out using JMP software v9. The phyloseq and vegan packages in $\mathrm{R}$ were used to calculate alpha- and beta-diversity and perform analyses and to produce two-dimensional Principal Coordinates Analysis plots using weighted UniFrac ${ }^{52}$ distances from the relative abundances of taxa for each sample. For beta-diversity analysis, the betadisper function in the vegan package was used to test for multivariate homogeneity of group dispersions using a permutational approach $^{53}$. PERMANOVA (in R) was used to determine differences (based on weighted UniFrac distances) being treatments and time points. Bootstrapped $95 \%$ CIs for pairwise differences between groups were calculated in $\mathrm{R}$, to allow comparison of magnitude of these pairwise differences.

Data availability. All sequencing information and data that support the findings of this study have been deposited in the European Nucleotide Archive (ENA) database with the accession codes PRJEB9900 and PRJEB13609. The authors declare that all other data are contained within the article and its Supplementary Files.

\section{References}

1. Low-Décarie, E. et al. Community rescue in experimental metacommunities. Proc. Natl Acad. Sci. USA 112, 14307-14312 (2015).

2. Norberg, J., Urban, M. C., Vellend, M., Klausmeier, C. A. \& Loeuille, N. Eco-evolutionary responses of biodiversity to climate change. Nat. Clim. Chang. 2, 747-751 (2012)

3. Antonovics, J. Plant population biology at the crossroads. Input from population genetics. Syst. Bot. 1, 234-245 (1976).

4. Schoener, T. W. The newest synthesis: understanding the interplay of evolutionary and ecological dynamics. Science 331, 426-429 (2011).

5. Hairston, N. G., Ellner, S. P., Geber, M. A., Yoshida, T. \& Fox, J. A. Rapid evolution and the convergence of ecological and evolutionary time. Ecol. Lett. 8, 1114-1127 (2005).

6. Ellner, S. P., Geber, M. A. \& Hairston, N. G. Does rapid evolution matter? Measuring the rate of contemporary evolution and its impacts on ecological dynamics. Ecol. Lett. 14, 603-614 (2011).

7. Pantel, J. H., Duvivier, C. \& Meester, L. D. Rapid local adaptation mediates zooplankton community assembly in experimental mesocosms. Ecol. Lett. 18, 992-1000 (2015)

8. Lawrence, D. et al. Species interactions alter evolutionary responses to a novel environment. PLoS Biol. 10, e1001330 (2012).

9. Yoshida, T., Jones, L. E., Ellner, S. P., Fussmann, G. F. \& Hairston, Jr N. G Rapid evolution drives ecological dynamics in a predator-prey system. Nature 424, 303-306 (2003).

10. Zhang, Q. G. \& Buckling, A. Antagonistic coevolution limits population persistence of a virus in a thermally deteriorating environment. Ecol. Lett. 14, 282-288 (2011).

11. McClean, D. et al. Single gene locus changes perturb complex microbial communities as much as apex predator loss. Nat. Commun. 6, 8235 (2015)

12. Johnson, M. T., Vellend, M. \& Stinchcombe, J. R. Evolution in plant populations as a driver of ecological changes in arthropod communities. Philos. Trans. R Soc. Lond. B Biol. Sci. 364, 1593-1605 (2009).

13. Harmon, L. J. et al. Evolutionary diversification in stickleback affects ecosystem functioning. Nature 458, 1167-1170 (2009).

14. Bassar, R. D. et al. Direct and indirect ecosystem effects of evolutionary adaptation in the Trinidadian guppy (Poecilia reticulata). Am. Nat. 180, 167-185 (2012).

15. Farkas, T. E., Mononen, T., Comeault, A. A., Hanski, I. \& Nosil, P. Evolution of camouflage drives rapid ecological change in an insect community. Curr. Biol. 23, 1835-1843 (2013).

16. Terhorst, C. P., Lennon, J. T. \& Lau, J. A. The relative importance of rapid evolution for plant-microbe interactions depends on ecological context. Proc. Biol. Sci. 281, 20140028 (2014).

17. Bailey, M. J., Lilley, A. K., Thompson, I. P., Rainey, P. B. \& Ellis, R. J. Site directed chromosomal marking of a fluorescent pseudomonad isolated from the phytosphere of sugar beet; Stability and potential for marker gene transfer. Mol. Ecol. 4, 755-763 (1995)

18. Gómez, P. \& Buckling, A. Real-time microbial adaptive diversification in soil. Ecol. Lett. 16, 650-655 (2013).

19. Buckling, A. \& Rainey, P. B Antagonistic coevolution between a bacterium and a bacteriophage. Proc. Biol. Sci. 269, 931-936 (2002).

20. Gómez, P. \& Buckling, A. Bacteria-phage antagonistic coevolution in soil. Science 332, 106-109 (2011).

21. Gómez, P., Ashby, B. \& Buckling, A. Population mixing promotes arms race host-parasite coevolution. Proc. Biol. Sci. 282, 20142297 (2015).

22. Caporaso, J. G. et al. QIIME allows analysis of high-throughput community sequencing data. Nat. Methods 7, 335-336 (2010).

23. Hutchinson, G. E. Population studies: animal ecology and demography concluding remarks. Cold Spring Harb. Symp. Quant. Biol. 22, 415-427 (1957).

24. Trippe, K., McPhail, K., Armstrong, D., Azevedo, M. \& Banowetz, G. Pseudomonas fluorescens SBW25 produces furanomycin, a non-proteinogenic amino acid with selective antimicrobial properties. BMC Microbiol. 13, 1-10 (2013).

25. Haas, D. \& Keel, C. Regulation of antibiotic production in root-colonizing Pseudomonas spp. and relevance for biological control of plant disease. Annu. Rev. Phytopathol. 41, 117-153 (2003).

26. Chase, J. M. Community assembly: when should history matter? Oecologia 136, 489-498 (2003).

27. Thompson, J. A., Oliveira, R. A., Djukovic, A., Ubeda, C. \& Xavier, K. B. Manipulation of the Quorum Sensing Signal AI-2 affects the antibiotic-treated gut microbiota. Cell Rep. 10, 1861-1871 (2015)

28. Gómez, P. \& Buckling, A. Coevolution with phages does not influence the evolution of bacterial mutation rates in soil. ISME J. 7, 2242-2244 (2013).

29. Scanlan, P. D. et al. Coevolution with bacteriophages drives genome-wide host evolution and constrains the acquisition of abiotic-beneficial mutations. Mol. Biol. Evol. 32, 1425-1435 (2015). 
30. Crutsinger, G. M. et al. Plant genotypic diversity predicts community structure and governs an ecosystem process. Science 313, 966-968 (2006).

31. Connell, J. H. \& Slatyer, R. O. Mechanisms of succession in natural communities and their role in community stability and organization. Am. Nat. 111, 1119-1144 (1977).

32. Fukami, T. Historical contingency in community assembly: integrating niches, species pools, and priority effects. Annu. Rev. Ecol. Evol. Syst. 46, 1 (2015).

33. Shulman, M. J. et al. Priority effects in the recruitment of juvenile coral reef fishes. Ecology 64, 1508-1513 (1983).

34. Sutherland, J. P. Multiple stable points in natural communities. Am. Nat. 108, 859-873 (1974).

35. De Meester, L., Gómez, A., Okamura, B. \& Schwenk, K. The Monopolization Hypothesis and the dispersal-gene flow paradox in aquatic organisms. Acta Oecologica 23, 121-135 (2002)

36. Fukami, T., Beaumont, H. J., Zhang, X. X. \& Rainey, P. B. Immigration history controls diversification in experimental adaptive radiation. Nature 446, 436-439 (2007)

37. Loeuille, N. \& Leibold, M. A. Evolution in metacommunities: on the relative importance of species sorting and monopolization in structuring communities. Am. Nat. 171, 788-799 (2008).

38. Urban, M. C. \& De Meester, L. Community monopolization: local adaptation enhances priority effects in an evolving metacommunity. Proc. Biol. Sci. 276, 4129-4138 (2009).

39. De Meester, L., Vanoverbeke, J., Kilsdonk, L. J. \& Urban, M. C. Evolving perspectives on monopolization and priority effects. Trends Ecol. Evol. 31, 136-146 (2016)

40. Modi, S. R., Collins, J. J. \& Relman, D. A. Antibiotics and the gut microbiota. J. Clin. Invest. 124, 4212-4218 (2014)

41. King, E. O., Ward, M. K. \& Raney, D. E. Two simple media for the demonstration of pyocyanin and fluorescin. J. Lab. Clin. Med. 44, 301-307 (1954).

42. Luján, A. M., Gómez, P. \& Buckling, A. Siderophore cooperation of the bacterium Pseudomonas fluorescens in soil. Biol. Lett. 11, 20140934 (2015).

43. Lenski, R. E., Rose, M. R., Simpson, S. C. \& Tadler, S. C. Long-term experimental evolution in Escherichia-Coli.1. Adaptation and divergence during 2,000 generations. Am. Nat. 138, 1315-1341 (1991).

44. Zhang, X.-X. \& Rainey, P. B. Construction and validation of a neutrally-marked strain of Pseudomonas fluorescens SBW25. J. Microbiol. Methods 71, 78-81 (2007).

45. Caporaso, J. G. et al. Global patterns of 16S rRNA diversity at a depth of millions of sequences per sample. Proc. Natl Acad. Sci. USA 108, 4516-4522 (2011).

46. Martin, M. Cutadapt removes adapter sequences from high-throughput sequencing reads. EMBnet.journal 17, 10-12 (2011).

47. Reeder, J. \& Knight, R. Rapidly denoising pyrosequencing amplicon reads by exploiting rank-abundance distributions. Nat. Methods 7, 668-669 (2010).

48. Magoc, T. \& Salzberg, S. L. FLASH: fast length adjustment of short reads to improve genome assemblies. Bioinformatics 27, 2957-2963 (2011).

49. Edgar, R. C. Search and clustering orders of magnitude faster than BLAST. Bioinformatics 26, 2460-2461 (2010).
50. McDonald, D. et al. An improved Greengenes taxonomy with explicit ranks for ecological and evolutionary analyses of bacteria and archaea. ISME J. 6, 610-618 (2012).

51. Wang, Q., Garrity, G. M., Tiedje, J. M. \& Cole, J. R. Naive Bayesian classifier for rapid assignment of rRNA sequences into the new bacterial taxonomy. Appl. Environ. Microbiol. 73, 5261-5267 (2007).

52. Lozupone, C. \& Knight, R. UniFrac: a new phylogenetic method for comparing microbial communities. Appl. Environ. Microbiol. 71, 8228-8235 (2005).

53. Anderson, M. J. Distance-based tests for homogeneity of multivariate dispersions. Biometrics 62, 245-253 (2006).

\section{Acknowledgements}

We thank Gabriel Yvon-Durocher for the useful comments on the manuscript, and John Kenny for technical assistance in $16 \mathrm{~S}$ sequencing. The work was funded by BBSRC, AXA Research fund and NERC. P.G. was supported by a Marie Curie Intra-European Fellowship within the European Commission 7th Framework Program (PIEF-GA-2010-272945), and acknowledges the Spanish MINECO support (AGL2014-59556-R). A.B. was supported by the Royal Society (UK). L.D.M. acknowledges the KU Leuven Research Fund support PF/2010/07.

\section{Author contributions}

P.G. and A.B. conceived and designed the study. P.G. conducted the experiments. S.P., P.G. and A.B. analysed the data. S.P., X.L., L.L. and M.D.S. performed the bioinformatics analysis. P.G., A.B. and S.P. wrote the manuscript. L.D.M. and K.M. contributed to manuscript revisions.

\section{Additional information}

Supplementary Information accompanies this paper at http://www.nature.com/ naturecommunications

Competing financial interests: The authors declare no competing financial interests.

Reprints and permission information is available online at http://npg.nature.com/ reprintsandpermissions/

How to cite this article: Gómez, P. et al. Local adaptation of a bacterium is as important as its presence in structuring a natural microbial community. Nat. Commun. 7:12453 doi: 10.1038/ncomms12453 (2016)

This work is licensed under a Creative Commons Attribution 4.0 International License. The images or other third party material in this article are included in the article's Creative Commons license, unless indicated otherwise in the credit line; if the material is not included under the Creative Commons license users will need to obtain permission from the license holder to reproduce the material To view a copy of this license, visit http://creativecommons.org/licenses/by/4.0/

(C) The Author(s) 2016 